\title{
34. Bon usage des psychotropes dans la maladie d'Alzheimer et syndromes apparentés
}

\author{
(C) Springer-Verlag France 2010
}

\section{4}

\author{
Alternatives pharmacologiques : \\ les traitements spécifiques de la maladie d'Alzheimer \\ S. Gauthier \\ $M D, F R C P C$, centre McGill d'études sur le vieillissement, \\ institut universitaire en santé mentale Douglas, Montréal, Canada
}

Les troubles de l'humeur et du comportement font partie du syndrome démentiel. Les symptômes les plus fréquents, utilisant la nomenclature du Dr Philippe Robert, basée sur le Neuropsychiatric Inventory (Cummings et al, Neurology 41, 1374-82, 1994) sont : idées délirantes, apathie, hallucinations, désinhibition, agitation, irritabilité, dépression, comportement moteur aberrant, anxiété, comportement durant la nuit, euphorie, modification de l'appétit et des comportements alimentaires. À cette liste s'est ajouté deux items, vocalisations et comportements sexuels inappropriés, dont la validation est en cours dans plusieurs langues, dont le français (De Medeiros et al, Int Psychogeriatrics 2010 [sous presse]). Ces troubles de l'humeur et du comportement varient quelque peu d'une pathologie à l'autre : les hallucinations et idées délirantes associées sont précoces et fréquentes dès le début d'une démence à corps de Lewy; dépression et apathie sont précoces dans la maladie d'Alzheimer. La sévérité d'un symptôme peut fluctuer dans le temps et un symptôme tel que l'agitation peut s'améliorer spontanément, nécessitant une réévaluation périodique du traitement. Il y a actuellement un effort soutenu pour aider les troubles de l'humeur et du comportement avec des approches non pharmacologiques (Ballard et al, Nature Reviews, Neurology 5, 245-55, 2009). Une meilleure formation des aidants et des modifications de l'environnement du malade permettent souvent de diminuer l'impact des symptômes. L'autre changement en cours est de trouver des alternatives aux neuroleptiques comme premier choix pharmacologique à cause de leur toxicité au point de vue cérébrovasculaire et mortalité et du peu d'efficacité démontré dans des essais cliniques randomisés. Malheureusement les preuves d'efficacité pour les médicaments alternatifs (antidépresseurs, antiépileptiques, inhibiteurs de l'acetylcholinesterase [IAChE], memantine) sont encore à un stade préliminaire et leur utilisation demande une évaluation individuelle et continue (Gauthier et al, Int Psychogeriatrics 22, 346-72, 2010). Les règles d'utilisation actuelles pour médications ciblant un comportement ne répondant pas à une approche non pharmacologique sont : 1) faire l'essai d'un IAChE pour hallucinations visuelles associées à une démence à début récent ; 2) traiter avec un antidépresseur non tricycliques s'il y a dépression, avant de donner un IAChE ; 3) faire l'essai de la mémantine s'il y a agitation et agressivité ; 4) faire l'essai de la trazodone en fin de journée ou soirée s'il y a symptômes associés au crépuscule ; 5) si un neuroleptique est absolument nécessaire administrer la dose la plus petite pour obtenir un effet thérapeutique et cesser dès que possible. 